\title{
PEDOT Polymer Film Based Counter Electrodes for Pt-free Dye- Sensitized Solar Cells
}

\author{
Jeong Kwon ${ }^{1}$ and Jong Hyeok Park ${ }^{1,2, *}$ \\ ${ }^{1}$ School of Chemical Engineering, Sungkyunkwan University, Suwon 440-746, Republic of Korea \\ ${ }^{2}$ SKKU Advanced Institute of Nanotechnology, Sungkyunkwan University, Suwon 440-746, Republic of Korea
}

\begin{abstract}
:
Poly (3,4-ethylenedioxythiophene) (PEDOT) polymers with different electric conductivities were synthesized directly on a FTO substrate via a chemical polymerization method and applied as a platinum (Pt)-free counter electrode for dye-sensitized solar cells (DSSCs). The catalytic properties of the PEDOT as a function of electrical conductivity were studied using cyclic voltammograms, J-V measurements and impedance spectroscopy. The PEDOT counter electrode with around $340 \mathrm{~S} /$ $\mathrm{cm}$ conductivity exhibited the best performance as a counter electrode for tri-iodide reduction. The ability to modulate catalytic activity of PEDOT with changes in conductivity shows one of promising routes for developing new counter electrode of Pt-free DSSCs.
\end{abstract}

Keywords : Dye-sensitized solar cells; PEDOT; Counter electrode; Pt-free

Received June 11, 2013 : Accepted August 14, 2013

\section{Introduction}

Dye-sensitized solar cells (DSSCs) with a mesoporous $\mathrm{TiO}_{2}$ film, sensitizers, iodine/iodide electrolyte, and platinum $(\mathrm{Pt})$ as a counter electrode have attracted the scientific and technological interest of researchers as a high-efficiency and low-cost alternative to commercialized inorganic photovoltaic devices. ${ }^{1-3)}$ Among the components in DSSCs, the counter electrode plays a crucial role in the reduction of iodide to tri-iodide ions. ${ }^{4}$ Until now, Pt on a FTO substrate has been employed as a counter electrode in DSSCs because of its extremely high catalytic ability. However, the need for high temperature sintering or high vacuum deposition processes to coat Pt on FTO substrate has spurred research into alternative counter electrode material. ${ }^{5-7)}$

In the past, many conducting polymers have been reported to replace Pt in DSSCs. For examples, vari-

*Corresponding author. Tel.: +82-31-290-7346

E-mail address: lutts@skku.edu ous research groups have attempted to replace Pt with conductive polymers such as PEDOT:PSS (poly $(3,4-$ ethylenedioxythiophene:poly (styrenesulfonate)), PEDOT, and PANI (polyaniline). ${ }^{8-12)}$ Typical conducting polymer counter electrode materials in DSSCs have been prepared on fluorine-doped tin oxide (FTO) coated glass substrates. Conducting polymers should have catalytic ability and charge transportation ability, simultaneously. So, tuning the electric conductivity of conducting polymers employed in DSSCs might be meaningful pathway to optimize their cell efficiencies.

In this paper, for the first time, we report on a conductivity controlled PEDOT counter electrode for a Pt-free counter electrode in DSSCs. The cell performances of DSSCs as a function of electric conductivity of PEDOT film were investigated. By tuning the electric conductivity of the conducting polymer, the DSSCs without Pt in the counter electrode showed a comparable cell efficiency with cells using a Pt/FTO counter electrode. These results will broaden the use of conductive polymers in DSSCs. 


\section{Experimental}

A PEDOT thin film was synthesized via the spincoating of a PEDOT oligomer solution ${ }^{12)}$ on top of FTO substrate, followed by thermal polymerization at $70^{\circ} \mathrm{C}$ for $20 \mathrm{~min}$. After finishing polymerization reaction, unreacted raw materials were removed by washing with methanol and drying (Fig. 1). Because polymerized PEDOT film can be well attached on a FTO substrate it survive from the washing process. By controlling EDOT monomer/oxidant ratios (1:5 to $5: 1)$, the resulting PEDOTs could have various electrical conductivities. To study the conductivity effect of PEDOT on the cell performance of Pt-free DSSCs, four different PEDOT films were prepared; $100 \mathrm{~S} /$ $\mathrm{cm}, 340 \mathrm{~S} / \mathrm{cm}, 580 \mathrm{~S} / \mathrm{cm}, 690 \mathrm{~S} / \mathrm{cm}$. For a comparison, a Pt/FTO counter electrode was simultaneously prepared by drop-casting a Pt precursor solution on an FTO glass substrate, followed by sintering at $400^{\circ} \mathrm{C}$ for 15 min. $^{12)}$

For fabrication of photoanodes, FTO glass substrates were cleaned with acetone, ethanol, and deionized water, then dried at $70^{\circ} \mathrm{C}$ for $30 \mathrm{~min}$. A thin $\mathrm{TiO}_{2}$ blocking layer on the cleaned FTO glass substrate was prepared from $40 \mathrm{mM}$ titanium tetrachloride $\left(\mathrm{TiCl}_{4}\right.$, Aldrich) treatment conducted at $70^{\circ} \mathrm{C}$ for $30 \mathrm{~min}$. The substrate was subsequently rinsed with ethanol several times. $\mathrm{TiO}_{2}$ nanoparticle electrodes $\left(\mathrm{TiO}_{2}\right.$ paste- $20 \mathrm{~nm}$ EnB Korea) were coated on the prepared FTO substrate previously via doctor blade method and sintered at $550^{\circ} \mathrm{C}$ for $30 \mathrm{~min}$. Then, the sintered $\mathrm{TiO}_{2}$ electrodes were immediately immersed in a $0.3 \mathrm{mM}$ solution of $\mathrm{N} 719$ dye in ethanol for $24 \mathrm{~h}$. Dye-adsorbed $\mathrm{TiO}_{2}$ photoanodes were removed from

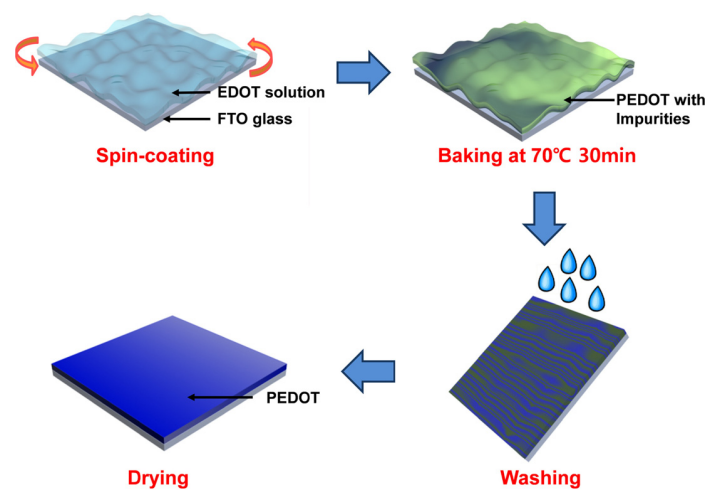

Fig. 1. Schematic diagram of the PEDOT counter electrode synthesis route. the dye solution, rinsed with ethanol, and then dried under nitrogen gas environment. The as-prepared $\mathrm{TiO}_{2}$ photoanodes and various counter electrodes were sandwiched using a $60 \mathrm{~mm}$-thick surlyn spacer (surlyn-1702, Dupont). The electrolyte composed of $0.6 \mathrm{M}$ butylmethylimidazolium iodide, $0.03 \mathrm{M} \mathrm{I}_{2}$, $0.1 \mathrm{M}$ guanidinium thiocyanate, and $0.5 \mathrm{M} 4$-tertbutylpyridine in a mixture of acetonitrile and valeronitrile (v/v, 85:15) was introduced into the sandwiched cell through a hole drilled in the counter electrode, and the hole was then sealed with a surlyn spacer and cover glass.

\section{Results and Discussion}

The electric conductivities of the synthesized PEDOT counter electrode used in Pt-free DSSCs were controlled by adjusting the molar ratio of the monomer to the oxidant or to the retardant. A schematic diagram of the PEDOT film is shown in Fig. 1. The cyclic voltammograms of $\mathrm{I}^{-} / \mathrm{I}_{3}{ }^{-}$redox on the various PEDOT films coated FTO glass substrate are shown in Fig. 2, illustrating that different PEDOT counter electrodes with different electric conductivities exhibited nearly the similar peak positions. However, the reduction peak current densities of the PEDOT counter electrodes were different as we expected. When $340 \mathrm{~S} / \mathrm{cm}$ PEDOT film represent the highest current density compared to other PEDOT samples, which represent more electrochemically active.

To investigate the influence of electric conductivity of PEDOT on DSSC cell performances, the

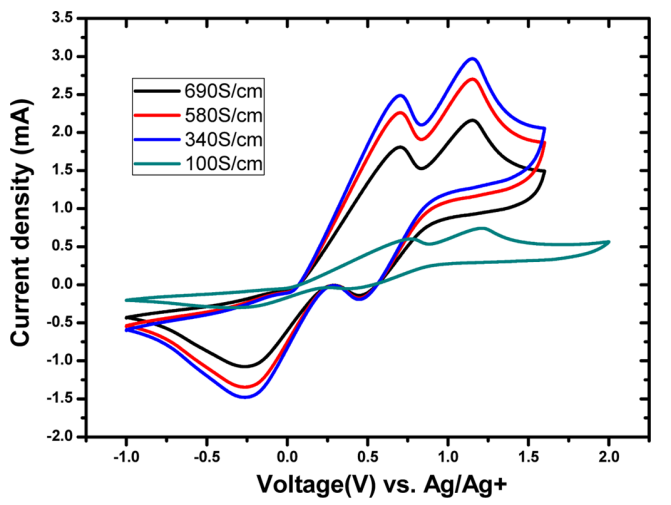

Fig. 2. Cyclic voltammograms for various PEDOT films with different electric conductivities measured at a scan rate of $50 \mathrm{mVs}^{\circ}{ }^{\circ 1}$ for the reduction of the iodine-based redox mediator. 


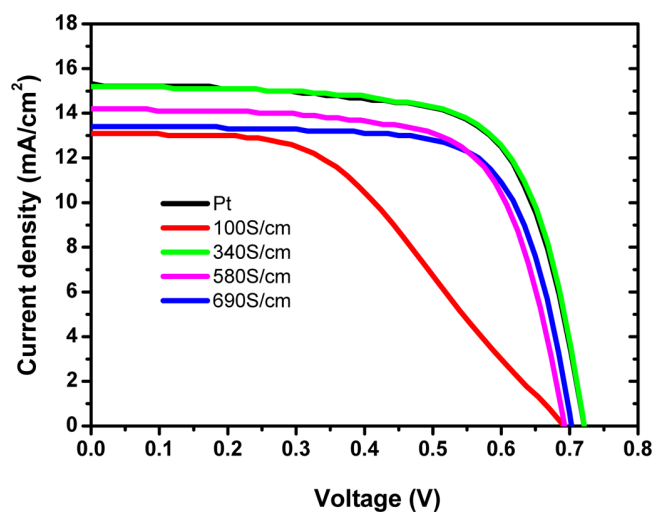

Fig. 3. Current-voltage characteristics of DSSCs made with various counter electrodes.

performance of such counter electrodes can be compared directly by fixing other parameters, such as the $\mathrm{TiO}_{2}$ layer thickness, dye loading, electrolyte, and conditions of cell assembly, as shown in Fig. 3. Current-voltage $(I-V)$ measurements of the DSSCs were investigated under AM 1.5 simulated solar light irradiation; a $300 \mathrm{~W}$ xenon lamp (Newport, USA) was used as a light source and a standard Si solar cell was employed for calibration. As we discussed in CV data, the performances of the DSSCs based on the PEDOT counter electrode were strongly dependent on the electric conductivity of the film. The DSSC with a PEDOT $(340 \mathrm{~S} / \mathrm{cm})$ counter electrode had the highest cell efficiency of $7.62 \%$ with fill factor of 0.70 and a $J_{s c}$ of $15.2 \mathrm{~mA} / \mathrm{cm}^{2}$, a $V_{o c}$ of $0.72 \mathrm{~V}$, which is comparable to using the Pt/TCO electrode (7.62\%).

In addition, EIS impedance (Fig. 4) was carried out under a light illumination condition through the use of an impedance analyzer (Zahner IM6). In general, a Nyquist plot of a DSSC consists of several semicircles, with the first semicircle in the high frequency region attributed to an interfacial charge transfer process at the counter electrode. ${ }^{13,14)}$ In the high-frequency semicircle which is corresponding to the performance of counter electrode, the values slightly increased with the PEDOT conductivity. In view point of charge transport, higher electric conductivity of PEDOT film can be helpful for the catalytic ability. However, PEDOT with higher electric conductivity contains less PEDOT content because of high concentration of ionic dopants from oxidants. With increasing electrical conductivity, the catalytic ability might be decreased gradually. As a result, the

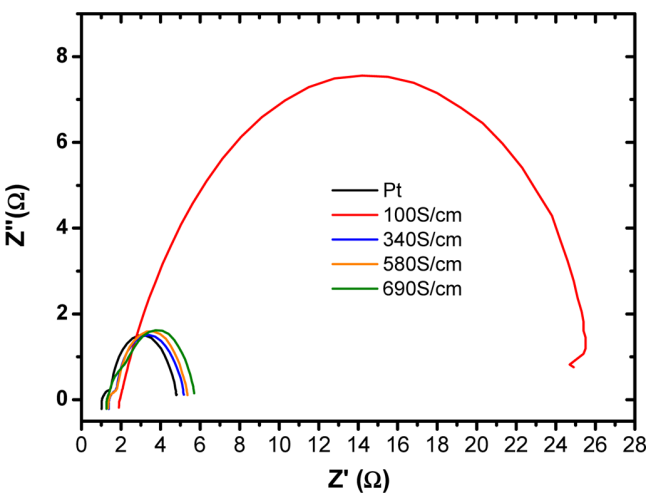

Fig. 4. Nyquist plots of DSSCs with different counter electrodes from the impedance measurement (cell area: $\left.0.1 \mathrm{~cm}^{2}\right)$.

DSSC with the $340 \mathrm{~S} / \mathrm{cm}$ PEDOT counter electrode shows the highest cell performances, which is similar to the $\mathrm{Pt} / \mathrm{TCO}$ cell.

\section{Conclusions}

In conclusions, various PEDOT film with different conductivities ranging from $100 \mathrm{~S} / \mathrm{cm}$ and $690 \mathrm{~S} / \mathrm{cm}$ were synthesized on a FTO glass substrate by a modified simple pre-solution/in situ polymerization method. The obtained efficiency was strongly dependent upon the conductivity values of PEDOT counter electrodes. Therefore, the electrical conductivity of the PEDOT film can be further optimized, the performance of a PEDOT based counter electrode can be improved, which eliminates the utility of Pt.

\section{Acknowledgment}

This work was supported by the New \& Renewable Energy R\&D Program of the Korea Institute of Energy Technology Evaluation and Planning(KETEP) grant funded by the Korea government Ministry of Trade, Industry \& Energy (No. 20123010010070).

\section{References}

1. B. O'Regan and M. Grätzel, Nature, 353, 737 (1991).

2. P. Wang, S.M. Zakeeruddin, P. Comte, I. Exnar, and M. Grätzel, J. Am. Chem. Soc., 125, 1166 (2003).

3. A. Yella, H.W. Lee, H.N. Tsao, C. Yi, A.K. Chandiran, M.K. Nazeeruddin, E.W.G. Diau, C.Y. Yeh, S.M. Zakeeruddin and M. Grätzel, Science, 334, 629 (2011). 
4. T.N. Murakami and M. Grätzel, Inorg. Chim. Acta, 361, 572 (2008).

5. N. Papageorgiou, Coord. Chem. Rev., 248, 1421 (2004).

6. R. Bashyam and P. Zelenary, Nature, 443, 63 (2006).

7. T.L. Hsieh, H.W. Chen, C.W. Kung, C.C. Wang, R. Vittal and K.C. Ho, J. Mater. Chem., 22, 5550 (2012).

8. M. Biancardo, K. West and F.C. Krebs, J. Photochem. Photobiology A: Chem., 187, 395 (2007).

9. T. Muto, M. Ikegami, K. Kobayashi and T. Miyasaka, Chem. Lett., 36, 804 (2007).

10. Q. Li, J. Wu, Q. Tang, Z. Lan, P. Li, J. Lin and L. Fan,
Electrochem. Commun., 10, 1299 (2008).

11. Y.H. Ha, N. Nikolov, S.K. Pollack, J. Mastrangelo, B.D. Martin and R. Shashidhar, Adv. Funct. Mater, 14, 615 (2004).

12. K.S. Lee, H.K. Lee, D.H. Wang, N.G. Park, J.Y. Lee, O.O. Park and J.H. Park, Chem. Commun., 46, 4505 (2010).

13. V. Ganapathy, B. Karunagaran and S.W. Rhee, ACS Appl. Mater. Interfaces, 3, 857 (2011).

14. K.S. Lee, Y. Jun and J.H. Park, Nano Lett., 12, 2233 (2012). 